\title{
The Realization of the Fuzzy Control for the Ultrasonic Determination of the Four-Axis Aircraft
}

\author{
Yu Yang ${ }^{1, ~ a, ~ X u e t a o ~ C h e n ~}{ }^{2, b}$ \\ ${ }^{1}$ School of Southwest Minzu University, Chengdu 610041, China \\ ${ }^{2}$ School of Southwest Minzu University, Chengdu 610041, China \\ a846995750@qq.com, b18200290471@163.com
}

\begin{abstract}
Fuzzy control theory is a kind of advanced control strategy based on fuzzy mathematics, language rule representation and advanced computer technology. This paper mainly introduces how to use ultrasonic sensor as the measurement variable delivery unit, and the fuzzy controller as the control unit to realize the height of the four-axis aircraft.
\end{abstract}

Keywords: Fuzzy Control, Ultrasonic Sensor, Membership Function.

\section{Fuzzy Control System}

Fuzzy Control is a combination of Fuzzy Mathematics and Control theory, it has been a very important branch of smart control.Fuzzy control is based on fuzzy sets and fuzzy linguistic rules, and the control rules are determined based on expert experience or on-site operator's experience and operation data. Using fuzzy control logic reasoning, it is difficult to establish an accurate mathematical model of complex objects to achieve effective control.The basic structure of Fuzzy Control System is shown in Fig.1 [2], compared with the Traditional Control System, the Fuzzy Control System replaces the Traditional Controller C with the Fuzzy Controller FC. So Fuzzy Controller is the core of Fuzzy Control System. In the fuzzy control system of the ultrasonic height setting of the four-axis aircraft, the deviation of the actual height of the aircraft and the ideal input height and the rate of change of the deviation are taken as the input of the fuzzy controller, and the height variation of the aircraft is taken as the output, ultrasonic sensors and motors serve as measuring transmission units and actuators, respectively, so that the aircraft is kept at a specified altitude (there is fluctuation). The Fuzzy Logic inference process of ultrasonic determination of aircraft is shown in Fig 2.

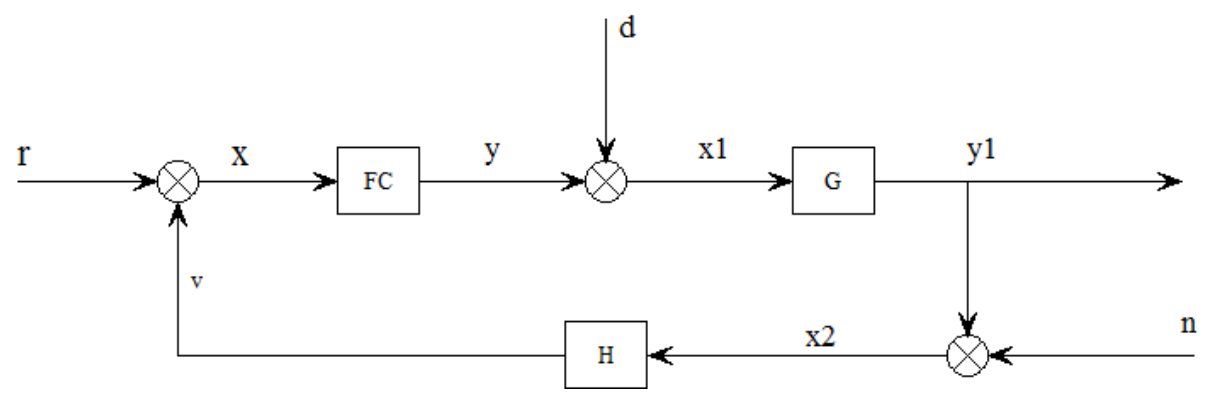

Fig. 1 The basic structure of a fuzzy control system 


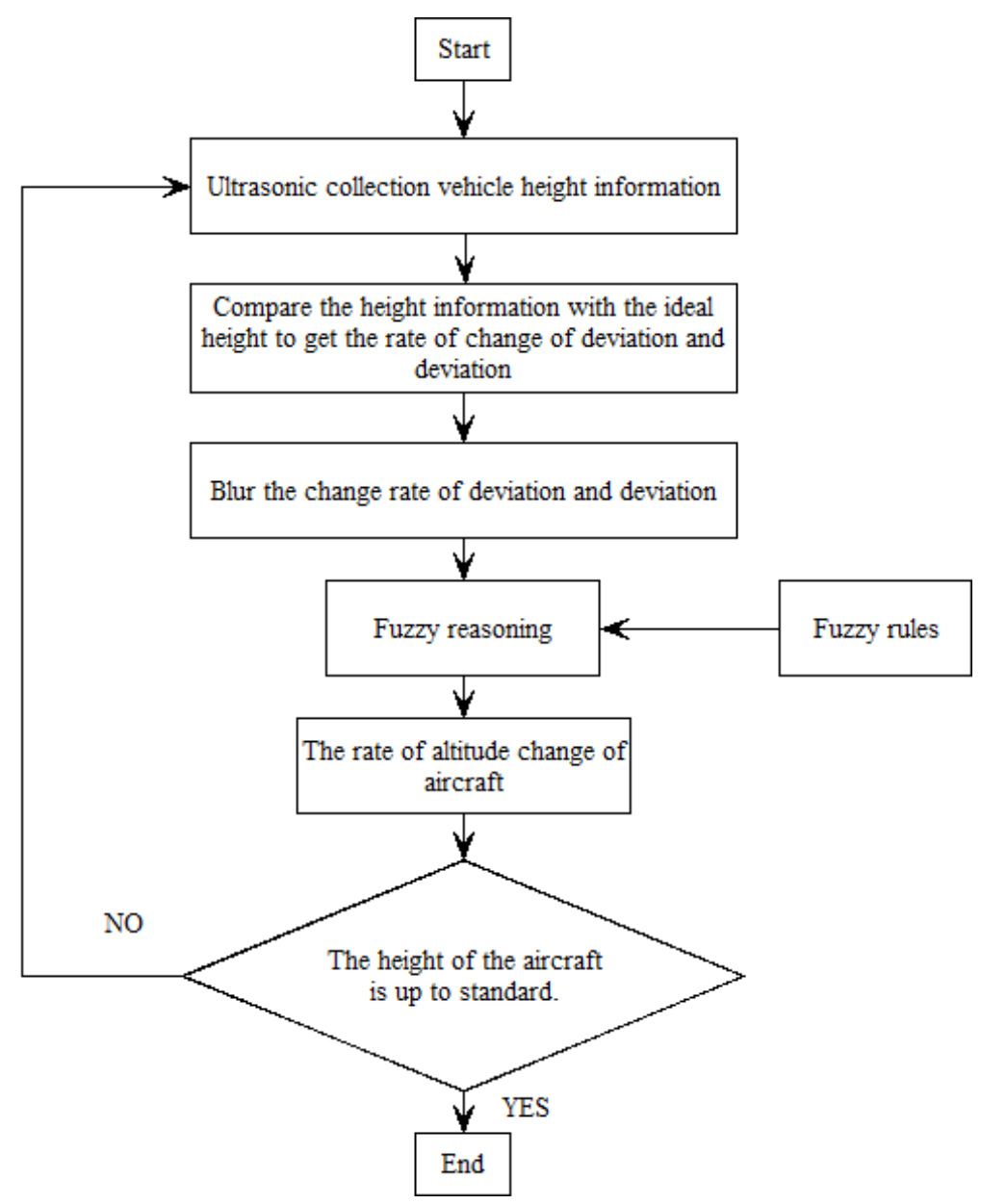

Fig. 2Fuzzy inference flow chart

\section{Fuzzy Controller Design}

\subsection{Fuzzy Controller Structure.}

The system structure of the fuzzy controller as shown in Fig. 3, working principle of the fuzzy controller, the first is the input digital signal through the blur $x(D / F)$ into fuzzy quantity, to be incorporated into fuzzy quantity with fuzzy rules of fuzzy reasoning module (R). through fuzzy reasoning conclusion, then the conclusion will be clearer $(\mathrm{F} / \mathrm{D})$ conversion into clear quantity $\mathrm{u}$, and then output to the next level to adjust the controlled object, to the satisfaction of the output results.

Aircraft by installing on the bottom of the ultrasonic sensors to detect the height of the aircraft and ground, our regulation was right in the vertical direction, and then concludes that aircraft high deviation $\mathrm{x} 1 \in[-10,10] \mathrm{cm}$ and deviation rate $\mathrm{x} 2 \in[-5,5] \mathrm{cm} / \mathrm{s}$, the fuzzy controller according to the height of $\mathrm{x} 1$ and $\mathrm{x} 2$ value choice of aircraft variation $\mathrm{v} \in[-30,50] \mathrm{cm} / \mathrm{s}$. Therefore, dual input - single output fuzzy controller is used for the purpose.

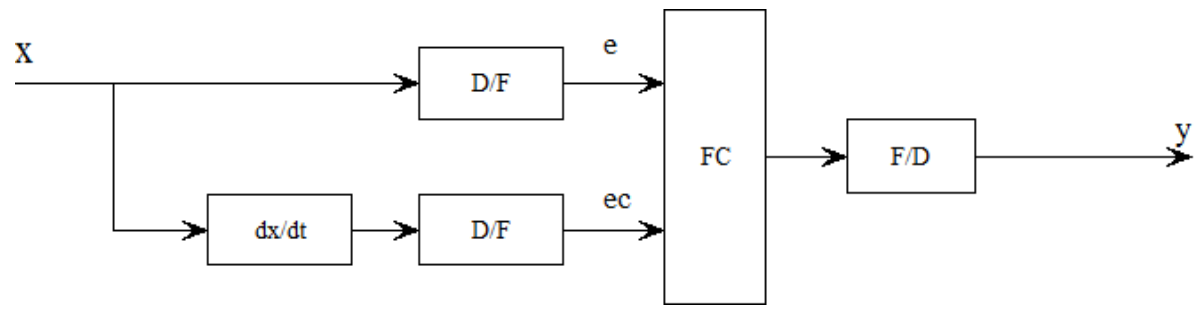

Fig. 3 The structure of the fuzzy controller 


\subsection{The Fuzziness of Input and Output.}

The process of converting the precise value of physical quantities into the value of language variables has achieved a precise amount of fuzziness.According to the actual situation, the classification of language variables is usually divided into $2 \sim 7$ subsection, the more the number of subsection, the higher the control accuracy, and the larger the calculation amount[3].

(1):According to the control requirements and the calculation amount, the height deviation $\mathrm{x} 1$ is divided into 5 subsections (PF,PN,M,NN,NF , and their membership functions are as follows.

$$
\begin{aligned}
& P F\left(x_{1}\right)=-\frac{1}{7} x_{1}-\frac{3}{7} \quad-10 \leq x 1 \leq-3 \\
& P N\left(x_{1}\right)= \begin{cases}\frac{1}{3} x_{1}+2 & -6 \leq x 1 \leq-3 \\
-\frac{1}{3} x_{1} & -3 \leq x 1 \leq 0\end{cases} \\
& P N\left(x_{1}\right)= \begin{cases}\frac{1}{3} x_{1}+1 & -3 \leq x 1 \leq 0 \\
-\frac{1}{3} x_{1}+1 & 0 \leq x 1 \leq 3\end{cases} \\
& P N\left(x_{1}\right)=\left\{\begin{array}{lc}
\frac{1}{3} x_{1} & 0 \leq x 1 \leq 3 \\
-\frac{1}{3} x_{1}+2 & 3 \leq x 1 \leq 6
\end{array}\right. \\
& P F\left(x_{1}\right)=\frac{1}{7} x_{1}-\frac{3}{7} \quad 3 \leq x 1 \leq 10
\end{aligned}
$$

(2):Divide the height deviation change rate $\mathrm{x} 2$ into 4 subsections $\{\mathrm{VPA}, \mathrm{PA}, \mathrm{NA}, \mathrm{VNA}$,$\} , and their$ membership functions are as follows.

$$
\begin{aligned}
& V P A\left(x_{1}\right)=-\frac{1}{3} x_{2}-\frac{2}{3} \quad-5 \leq x_{2} \leq-2 \\
& P A\left(x_{1}\right)= \begin{cases}-\frac{1}{2} x_{2}+\frac{1}{2} & -3 \leq x_{2} \leq-1 \\
-\frac{1}{2} x_{2}+\frac{1}{2} & -1 \leq x_{2} \leq 1\end{cases} \\
& N A\left(x_{1}\right)= \begin{cases}\frac{1}{2} x_{2}+\frac{1}{2} & -1 \leq x_{2} \leq 1 \\
-\frac{1}{2} x_{2}+1 & 1 \leq x_{2} \leq 3\end{cases} \\
& V N A\left(x_{1}\right)=\frac{1}{3} x_{2}-\frac{2}{3} \quad 3 \leq x_{2} \leq 10
\end{aligned}
$$

(3):Divide the height change of the aircraft $\mathrm{v}$ into 5 subsections $\{\mathrm{FGR}, \mathrm{SGR}, \mathrm{S}, \mathrm{SGD}, \mathrm{FGD}\}$, and their membership functions are as follows.

$$
\begin{aligned}
& F G R\left(x_{1}\right)=-\frac{1}{21.5} y-\frac{1.7}{4.3} \quad-30 \leq y \leq-8.5 \\
& \operatorname{SGR}\left(x_{1}\right)=\left\{\begin{array}{lc}
\frac{1}{10} y+1.85 & -18.5 \leq y \leq-8.5 \\
-\frac{1}{10} \mathrm{y}+0.15 & -8.5 \leq y \leq 1.5
\end{array}\right. \\
& S\left(x_{1}\right)=\left\{\begin{array}{lr}
\frac{1}{10} y+0.85 & -8.5 \leq y \leq 1.5 \\
-\frac{1}{13.5} y+\frac{10}{9} & 1.5 \leq y \leq 15
\end{array}\right. \\
& S G D\left(x_{1}\right)=\left\{\begin{array}{lc}
\frac{1}{13.5} y-\frac{1}{9} & 1.5 \leq y \leq 15 \\
-\frac{1}{13.5} y+\frac{19}{9} & 15 \leq y \leq 28.5
\end{array}\right. \\
& P F\left(x_{1}\right)=\frac{1}{35} y-\frac{\dot{3}}{7} \quad 15 \leq y \leq 50
\end{aligned}
$$

\subsection{The Establishment of Fuzzy Rules.}

According to the control requirements, the following 20 fuzzy rules can be formulated:

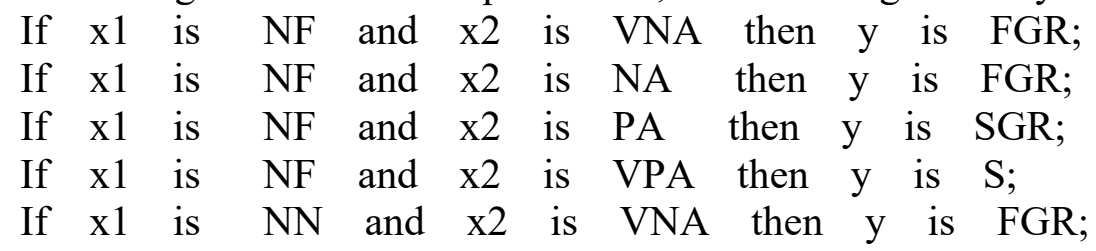




\begin{tabular}{|c|c|c|c|c|c|c|c|c|c|c|}
\hline$f \quad x 1$ & S & $\mathrm{N}$ & ad & X2 & is & NA & & & is & \\
\hline $\mathrm{x} 1$ & is & $\mathrm{NN}$ & an & $\mathrm{x} 2$ & is & PA & then & $\mathrm{y}$ & is & $\mathrm{S}$; \\
\hline x1 & is & $\mathrm{NN}$ & and & x2 & is & VPA & then & $\mathrm{y}$ & is & $\mathrm{S}$; \\
\hline $\mathrm{x} 1$ & is & M & and & $\mathrm{x} 2$ & is & VNA & then & $\mathrm{y}$ & is & SG \\
\hline x1 & is & $\mathrm{M}$ & & $\mathrm{x} 2$ & is & NA & then & $\mathrm{y}$ & is & $\mathrm{S}$; \\
\hline $\mathrm{x} 1$ & is & & & $x 2$ & is & PA & n & $\mathrm{y}$ & is & $\mathrm{S}$ \\
\hline $\mathrm{x} 1$ & is & M & & $\mathrm{x} 2$ & is & VPA & & $\mathrm{y}$ & is & $\mathrm{S}$; \\
\hline $\mathrm{x} 1$ & is & PN & & x2 & is & VNA & then & $\mathrm{y}$ & is & $\mathrm{S}$; \\
\hline $\mathrm{x} 1$ & is & PN & & $x^{\prime}$ & is & NA & then & $\mathrm{y}$ & is & FGD; \\
\hline x1 & is & $\mathrm{PN}$ & & $\mathrm{x} 2$ & is & PA & then & $\mathrm{y}$ & is & FGD; \\
\hline $\mathrm{x} 1$ & is & PN & & $\mathrm{x} 2$ & is & VPA & $n$ & $\mathrm{y}$ & is & FGR; \\
\hline x1 & is & PF & & $\mathrm{x} 2$ & is & VNA & then & $\mathrm{y}$ & is & $\mathrm{S}$; \\
\hline $\mathrm{x} 1$ & is & PF & & $\alpha$ & is & NA & then & $\mathrm{y}$ & is & SGD \\
\hline $\mathrm{x} 1$ & is & $\mathrm{PF}$ & & $x 2$ & is & PA & & $\mathrm{y}$ & is & FGD; \\
\hline $\mathrm{x} 1$ & is & PF & & $\mathrm{x} 2$ & is & VPA & & $y$ & is & FGL \\
\hline
\end{tabular}

\section{MATLAB Simulation}

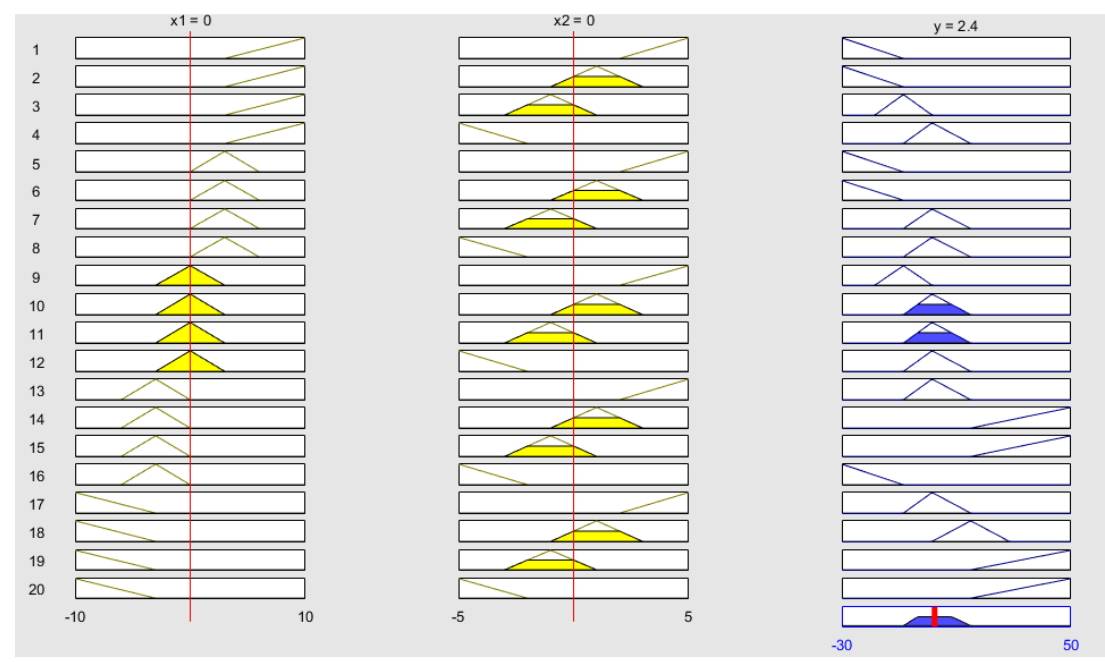

Fig. 4 Controller Rule

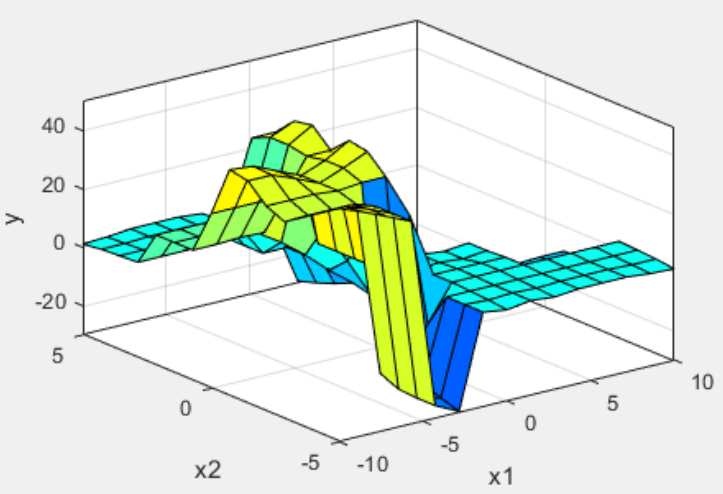

Fig. 5 Controller FIS output

\section{Summary}

By integrating the ultrasonic sensor with the four-axis aircraft, we can obtain the height information of the aircraft, and realize the ideal flying height by using the fuzzy control idea. This design realizes the effective control of a clear and complicated physical quantity. 


\section{References}

[1]. Yu Haisheng, Ding Junhang, Pan Songfeng, Wu Herong, Microcomputer Control Technology (2nd Edition). Tsinghua Press,2017.

[2]. Huang Weihua, Fuzzy Control System and Application - Beijing: Publishing House of Electronics Industry, (2012.11).

[3]. [3] Wang Dong, Autonomous obstacle avoidance for mobile robots based on multiple ultrasonic ranging and fuzzy control[D]. Ocean University of China,(2008). 